\title{
PRODUCT OF STATISTICAL PROBABILITY CONVERGENCE AND ITS APPLICATIONS TO KOROVKIN-TYPE THEOREM
}

\author{
BIDU BHUSAN JENA AND SUSANTA KUMAR PAIKRAY
}

Received 08 September, 2019

\begin{abstract}
In the present work, we introduce and study the notion of statistical probability convergence for sequences of random variables as well as the concept of statistical convergence for sequences of real numbers, which are defined over a Banach space via product of deferred Cesàro and deferred Nörlund summability means. We first establish a theorem presenting a connection between them. Based upon our proposed method, we then prove a Korovkin-type approximation theorem with algebraic test functions for a sequence of random variables on a Banach space and demonstrate that our theorem effectively extends and improves most (if not all) of the previously existing results (in classical as well as statistical versions). Finally, an illustrative example is presented here by means of the generalized Meyer-König and Zeller operators for a positive sequence of random variables in order to demonstrate that our established theorem is stronger than its traditional and statistical versions.
\end{abstract}

2010 Mathematics Subject Classification: 40A05; 40G15; 41A36

Keywords: statistical convergence, statistical probability convergence product of deferred Cesàro and deferred Nörlund statistical probability convergence, positive linear operators, sequence of random variables, Banach space, Korovkin-type theorems

\section{INTRODUCTION AND MOTIVATION}

In the study of sequence spaces, the classical convergence has got numerous applications where the convergence of a sequence requires that almost all elements are to satisfy the convergence condition. This means that, all of the elements of the sequence need to be in an arbitrarily small neighborhood of the limit. However, such restriction is relaxed in statistical convergence, where the validity of the convergence condition is achieved only for a majority of elements. Subsequently, the notion of statistical convergence was introduced by Fast [6] and Steinhaus [27]. Recently, statistical probability convergence has been a dynamic research area due to the fact that it is more general than the statistical convergence as well as the classical convergence. Moreover, such theory is discussed in the study of Fourier Analysis, Number Theory and Approximation Theory. For more details, see [9], [7] and [19]. 
Let $\mathbb{N}$ be the set of natural numbers and let $K \subseteq \mathbb{N}$. Also let

$$
K_{n}=\{k: k \leqq n \quad \text { and } \quad k \in K\}
$$

and suppose that $\left|K_{n}\right|$ is the cardinality of $K_{n}$. Then the natural density $d(K)$ of $K$ is defined by

$$
d(K)=\lim _{n \rightarrow \infty} \frac{\left|K_{n}\right|}{n}=\lim _{n \rightarrow \infty} \frac{1}{n} \mid\{k: k \leqq n \quad \text { and } \quad k \in K\} \mid,
$$

provided that the limit exists.

A given real sequence $\left(x_{n}\right)$ is said to be statistically convergent to $L$ if, for each $\epsilon>0$, the set

$$
K_{\epsilon}=\left\{k: k \in \mathbb{N} \quad \text { and } \quad\left|x_{k}-L\right| \geqq \epsilon\right\}
$$

has zero natural density (see [6] and [27]). Thus, for each $\epsilon>0$, we have

$$
d\left(K_{\epsilon}\right)=\lim _{n \rightarrow \infty} \frac{\left|K_{\epsilon}\right|}{n}=\lim _{n \rightarrow \infty} \frac{1}{n} \mid\left\{k: k \leqq n \quad \text { and } \quad\left|x_{k}-L\right| \geqq \epsilon\right\} \mid=0 .
$$

In this case, we write

$$
\text { stat } \lim _{n \rightarrow \infty} x_{n}=L \text {. }
$$

In the year 2002, Móricz [13] introduced the fundamental idea of statistical Cesàro summability. Later on, Mohiuddine et al. [12] established some Korovkin-type approximation theorems based upon statistical Cesàro summability. Subsequently, Karakaya and Chishti [10] introduced and studied the concept of weighted statistical convergence and their definition was later modified by Mursaleen et al. [14]. Furthermore, the fundamental concept of the deferred Cesàro statistical convergence as well as of the statistically-deferred Cesàro summability and associated approximation theorems was introduced by Jena et al. [8]. Recently, Srivastava et al. [22] introduced the notion of deferred weighted statistical convergence and proved analogous approximation theorems. For several other recent developments in this direction, see (for example) [4], [15], [17], [22], [24], [23] and [25].

Recalling the probability theory, let $X_{n} \quad(n \in \mathbb{N})$ be a random variable defined on an event space $S$ with respect to a given class of events $\Delta$. Let $P: \Delta \rightarrow \mathbb{R}$ (where $\mathbb{R}$ is the set of real numbers) be a probability density function. Then we denote the sequence $X_{1}, X_{2}, X_{3}, \cdots$ of random variables by $\left\{X_{n}\right\}_{n \in \mathbb{N}}$.

From the practical point of view, the discussion of a random variable $X$ will be highly significant if it is known that there exists a real constant $c$ for which

$$
P(|X-c|<\epsilon)=1,
$$

where $\epsilon>0$ is sufficiently small, that is, it is nearly certain that the values of $X$ lie in a very small neighborhood of $c$. 
For a sequence $\left\{X_{n}\right\}_{n \in \mathbb{N}}$ of random variables, where each $X_{n}$ may not have the above property, it may happen that the aforesaid property (with respect to a real constant $c$ ) becomes more and more distinguished as $n$ gradually increases and then the question of existence of such a real constant $c$ will be answered by a notion of probability convergence (that is, convergence in probability) of the sequence $\left\{X_{n}\right\}_{n \in \mathbb{N}}$.

Let $\left\{X_{n}\right\}_{n \in \mathbb{N}}$ be a sequence of random variables, where each $X_{n}$ is defined on the same event space $S$, with respect to a given class of subsets (of $S$ ) as the class $\Delta$ of events and a given probability function $P: \Delta \rightarrow \mathbb{R}$. The sequence $\left\{X_{n}\right\}_{n \in \mathbb{N}}$ is said to be statistically probability convergent (or statistically convergent in probability) to a random variable $X$ (where $X: S \rightarrow \mathbb{R}$ ) if, for any $\epsilon>0$ and $\delta>0$, we have

$$
\lim _{n \rightarrow \infty} \frac{1}{n} \mid k: k \leqq n \quad \text { and } \quad P\left(\left|X_{n}-X\right| \geqq \epsilon\right) \geqq \delta \mid=0
$$

or, equivalently,

$$
\lim _{n \rightarrow \infty} \frac{1}{n} \mid k: k \leqq n \quad \text { and } \quad 1-P\left(\left|X_{n}-X\right| \leqq \epsilon\right) \geqq \delta \mid=0 .
$$

In this case, we write

$$
\text { statP } \lim _{n \rightarrow \infty} P\left(\left|X_{n}-X\right| \geqq \epsilon\right)=0 \quad \text { or } \quad \text { stat }_{P} \lim _{n \rightarrow \infty} P\left(\left|X_{n}-X\right| \leqq \epsilon\right)=1 .
$$

We now show by means of the following example that every statistically convergent sequence is statistically probability convergent, but the converse is not necessarily true.

Example 1. Consider a probability density function of $X_{n}$ of the following form:

$$
f_{n}(x)= \begin{cases}\frac{1}{3} & \left(0<x<1 ; n=m^{2} \forall m \in \mathbb{N}\right) \\ 0 & \text { (otherwise) }\end{cases}
$$

and

$$
f_{n}(x)= \begin{cases}\frac{n x^{n-1}}{4^{n}} & \left(0<x<4 ; n \neq m^{2} \forall m \in \mathbb{N}\right) \\ 0 & \text { (otherwise) } .\end{cases}
$$

Let $0<\epsilon, \delta<1$. Then

$$
P\left(\left|X_{n}-4\right| \geqq \epsilon\right)=\left\{\begin{array}{cc}
1 & \left(n=m^{2} \forall m \in \mathbb{N}\right) \\
1-P\left(\left|X_{n}-4\right|<\epsilon\right) & \\
=\left(1-\frac{\epsilon}{4}\right)^{n} & \left(n \neq m^{2} \forall m \in \mathbb{N}\right) .
\end{array}\right.
$$


This implies that

$$
\begin{aligned}
\lim _{n \rightarrow \infty} & \frac{1}{n} \mid\left\{k: k \leqq n \quad \text { and } \quad P\left(\left|X_{n}-4\right| \geqq \epsilon\right) \geqq \delta\right\} \mid \\
& \leqq \lim _{n \rightarrow \infty} \frac{1}{n}\left|\left\{1^{2}, 2^{2}, 3^{2}, \cdots, n^{2}\right\}\right|=0 .
\end{aligned}
$$

Clearly, it is neither statistically convergent nor ordinarily convergent, while it is statistically probability convergent to 4 .

Quite recently, Srivastava et al. ([20] and [21]) first introduced and studied the fundamental idea of deferred Cesàro statistical probability convergence as well as deferred weighted statistical probability convergence of a sequence of random variables as follows.

Let $\left\{X_{n}\right\}_{n \in \mathbb{N}}$ be a sequence of random variables, where each $\left(X_{n}\right)$ is defined on the same event space $S$ with respect to a given class of subsets (of $S$ ) as the class $\Delta$ of events and a given probability function $P: \Delta \rightarrow \mathbb{R}$. A given sequence $\left\{X_{n}\right\}_{n \in \mathbb{N}}$ is said to be deferred Cesàro statistically probability convergent to a random variable $X$ (where $X: S \rightarrow \mathbb{R}$ ), if for every $\delta>0$ and $\epsilon>0$, the set

$$
\left\{k: a_{n}<k \leqq b_{n} \quad \text { and } \quad P\left(\left|X_{n}-X\right| \geqq \epsilon\right) \geqq \delta\right\}
$$

has natural density zero, that is,

$$
\lim _{n \rightarrow \infty} \frac{1}{b_{n}-a_{n}} \mid\left\{k: a_{n}<k \leqq b_{n} \quad \text { and } \quad P\left(\left|X_{n}-X\right| \geqq \epsilon\right) \geqq \delta\right\} \mid=0,
$$

In this case, we write

$$
\text { stat }_{\text {DCP }} \lim _{n \rightarrow \infty} P\left(\left|X_{n}-X\right| \geqq \epsilon\right)=0 .
$$

Similarly, a given sequence $\left\{X_{n}\right\}_{n \in \mathbb{N}}$ is said to be deferred weighted statistically probability convergent to a random variable $X$ (where $X: S \rightarrow \mathbb{R}$ ), if for every $\delta>0$ and $\epsilon>0$, the set

$$
\left\{m: m \leqq \mathcal{R}_{n} \quad \text { and } \quad p_{m} q_{m} P\left(\left|X_{n}-X\right| \geqq \epsilon\right) \geqq \delta\right\}
$$

has natural density zero, that is,

$$
\lim _{n \rightarrow \infty} \frac{1}{\mathcal{R}_{n}} \mid\left\{m: m \leqq \mathcal{R}_{n} \quad \text { and } \quad p_{m} q_{m} P\left(\left|X_{n}-X\right| \geqq \epsilon\right) \geqq \delta\right\} \mid=0 .
$$

In this case, we write

$$
\text { stat }_{\mathrm{DN} \mathrm{P}} \lim _{n \rightarrow \infty} p_{m} q_{m} P\left(\left|X_{n}-X\right| \geqq \epsilon\right)=0 .
$$

Several researchers have worked on extending or generalizing the Korovkin-type approximation theorems in many different ways and under several different settings, including function spaces, abstract Banach lattices, Banach algebras, Banach spaces, and so on. This theory is very useful in Real Analysis, Functional Analysis, Harmonic 
Analysis, Measure Theory, Probability Theory and Summability Theory. In the year 2018, Jena et al. [8] introduced statistically-deferred Cesàro summability for single sequences in Korovkin-type approximation theorems. Recently, Paikray et al. [16] established a Korovkin-type theorem based upon the $(p, q)$-integers for statisticallydeferred Cesàro summability mean. Subsequently, Dutta et al. [5] demonstrated the Korovkin theorem on $\mathcal{C}[0, \infty)$ by using the test functions $1, e^{-x}$ and $e^{-2 x}$ via the deferred Cesàro mean. In another recent work, Srivastava et al. [24] made use of the notion of the deferred Nörlund statistical convergence and accordingly proved a Korovkin-type approximation theorem.

Motivated essentially by the above-mentioned investigations and results, we first introduce here the notion of the product of deferred Cesàro and deferred Nörlund statistical convergence of real sequences, and then for the statistical probability convergence of sequences of random variables. Moreover, we establish an inclusion relation between them. Furthermore, based upon our proposed methods, we prove a new Korovkin-type approximation theorem with algebraic test functions for positive sequences of random variables over a Banach space and demonstrate that our result is a non-trivial extension of some well-established ordinary and statistical versions of several known results.

\section{PRELIMINARIES AND DEFINITIONS}

Let $\left(a_{n}\right)$ and $\left(b_{n}\right)$ be sequences of non-negative integers such that, (i) $a_{n}<b_{n}$ and (ii) $\lim _{n \rightarrow \infty} b_{n}=\infty$, then the deferred Cesàro (DC) mean is defined by (see, Agnew [1], p. 414),

$$
\begin{aligned}
\sigma_{n} & =\frac{x_{a_{n}+1}+x_{a_{n}+2}+x_{a_{n}+3}+\ldots+x_{b_{n}}}{b_{n}-a_{n}} \\
& =\frac{1}{b_{n}-a_{n}} \sum_{k=a_{n}+1}^{b_{n}} x_{k} .
\end{aligned}
$$

It is well known that, deferred Cesàro (DC) mean is regular under conditions (i) and (ii) (see, Agnew [1]).

Similarly, suppose that $\left(p_{n}\right)$ be a sequence of non-negative real numbers such that

$$
\mathcal{P}_{n}=\sum_{m=a_{n}+1}^{b_{n}} p_{b_{n}-m},
$$

then the deferred Nörlund mean $D\left(N, p_{n}\right)$ is defined by (see [24])

$$
t_{n}=\frac{1}{\mathcal{P}_{n}} \sum_{m=a_{n}+1}^{b_{n}} p_{b_{n}-m} x_{m} .
$$


Also, it is well known that, $D\left(N, p_{n}\right)$ is regular under the above-mentioned conditions (i) and (ii) (see, for details, Agnew [1]).

We now define the product of deferred Cesàro (DC) and deferred Nörlund (DN) means

$$
\begin{aligned}
\Lambda_{n}=(\sigma t)_{n} & =\frac{1}{\left(b_{n}-a_{n}\right)} \sum_{m=a_{n}+1}^{b_{n}}\left(t_{m}\right) \\
& =\frac{1}{\left(b_{n}-a_{n}\right)} \sum_{m=a_{n}+1}^{b_{n}} \frac{1}{\mathcal{P}_{m}} \sum_{v=a_{m}+1}^{b_{m}} p_{b_{m}-v} x_{v},
\end{aligned}
$$

and that a sequence $\left(\Lambda_{n}\right)$ is summable to $L$ by the deferred Cesàro (DC) and deferred Nörlund $(\mathrm{DN})$ product $[\mathrm{D}(\mathrm{CN})]$ means if,

$$
\lim _{n \rightarrow \infty} \Lambda_{n}=L .
$$

Also, assume that the $[\mathrm{D}(\mathrm{CN})]$ product mean is regular.

Let us now introduce the following definitions which will be needed in connection with our proposed investigation here.

Definition 1. Let $\left(a_{n}\right)$ and $\left(b_{n}\right)$ be sequences of non-negative integers, and let $\left(p_{n}\right)$ be the sequence of non-negative real numbers. A real sequence $\left\{x_{n}\right\}_{n \in \mathbb{N}}$ is said to be deferred Cesàro and deferred Nörlund $[\mathrm{D}(\mathrm{CN})]$ statistically convergent to $L$ if, for each $\epsilon>0$, the set given by

$$
\left\{m: m \leqq\left(b_{n}-a_{n}\right) \mathcal{P}_{n} \quad \text { and } \quad p_{b_{n}-m}\left|x_{m}-L\right| \geqq \epsilon\right\}
$$

has its natural density zero, that is,

$$
\lim _{n \rightarrow \infty} \frac{1}{\left(b_{n}-a_{n}\right) \mathcal{P}_{n}} \mid\left\{m: m \leqq\left(b_{n}-a_{n}\right) \mathcal{P}_{n} \quad \text { and } \quad p_{b_{n}-m}\left|x_{m}-L\right| \geqq \epsilon\right\} \mid=0 .
$$

In this case, we write

$$
\operatorname{stat}_{\mathrm{D}(\mathrm{CN})} \lim x_{n}=L .
$$

Definition 2. Let $\left(a_{n}\right)$ and $\left(b_{n}\right)$ be sequences of non-negative integers and let $\left(p_{n}\right)$ be the sequence of non-negative real numbers. Suppose also that $\left\{X_{n}\right\}_{n \in \mathbb{N}}$ is a sequence of random variables, where each $\left(X_{n}\right)$ is defined on the same event space $S$ with respect to a given class $\Delta$ of subsets of the event space $S$ and a given probability density function $P: \Delta \rightarrow \mathbb{R}$. A given sequence $\left\{X_{n}\right\}_{n \in \mathbb{N}}$ is said to be deferred Cesàro and deferred Nörlund $[\mathrm{D}(\mathrm{CN}) \mathrm{P}]$ statistically probability convergent to a random variable $X$ (where $X: S \rightarrow \mathbb{R}$ ) if, for every $\delta>0$ and $\epsilon>0$, the set

$$
\left\{m: m \leqq\left(b_{n}-a_{n}\right) \mathcal{P}_{n} \quad \text { and } \quad p_{b_{n}-m} P\left(\left|X_{n}-X\right| \geqq \epsilon\right) \geqq \delta\right\}
$$


has its natural density equal to zero, that is,

$\lim _{n \rightarrow \infty} \frac{1}{\left(b_{n}-a_{n}\right) \mathcal{P}_{n}} \mid\left\{m: m \leqq\left(b_{n}-a_{n}\right) \mathcal{P}_{n} \quad\right.$ and $\left.\quad p_{b_{n}-m} P\left(\left|X_{n}-X\right| \geqq \epsilon\right) \geqq \delta\right\} \mid=0$ or, equivalently,

$$
\begin{aligned}
& \lim _{n \rightarrow \infty} \frac{1}{\left(b_{n}-a_{n}\right) \mathcal{P}_{n}} \mid\left\{m: m \leqq\left(b_{n}-a_{n}\right) \mathcal{P}_{n}\right. \\
& \text { and } \left.1-p_{b_{n}-m} P\left(\left|X_{n}-X\right| \leqq \epsilon\right) \geqq \delta\right\} \mid=0 .
\end{aligned}
$$

In this case, we write

$$
\operatorname{stat}_{\mathrm{D}(\mathrm{CN}) \mathrm{P}} \lim _{n \rightarrow \infty} p_{b_{n}-m} P\left(\left|X_{n}-X\right| \geqq \epsilon\right)=0
$$

or

$$
\operatorname{stat}_{\mathrm{D}(\mathrm{CN}) \mathrm{P}} \lim _{n \rightarrow \infty} p_{b_{n}-m} P\left(\left|X_{n}-X\right| \leqq \epsilon\right)=1 .
$$

We next present a theorem demonstrating that every deferred Cesàro and deferred Nörlund $[\mathrm{D}(\mathrm{CN})]$ statistically convergent sequence is deferred Cesàro and deferred Nörlund $[\mathrm{D}(\mathrm{CN}) \mathrm{P}]$ statistically probability convergent. However, the converse is not true.

Theorem 1. Let the sequence $\left\{x_{n}\right\}$ of constants be such that $\operatorname{stat}_{\mathrm{D}(\mathrm{CN})} x_{n} \rightarrow x$. Then, assuming it to be a random variable having a one-point distribution at that point, the sequence $\left\{X_{n}\right\}$ of random variables is such that

$$
\text { stat }_{\mathrm{D}(\mathrm{CN}) \mathrm{P}} X_{n} \rightarrow X \text {. }
$$

Proof. Let $\epsilon>0$ be any arbitrarily small positive real number. Then, by Definition 1 , we obtain

$$
\lim _{n \rightarrow \infty} \frac{1}{\left(b_{n}-a_{n}\right) \mathcal{P}_{n}} \mid\left\{m: m \leqq\left(b_{n}-a_{n}\right) \mathcal{P}_{n} \quad \text { and } \quad p_{b_{n}-m}\left|x_{m}-L\right| \geqq \epsilon\right\} \mid=0 .
$$

We now let $\delta>0$, so that the set

$$
\left\{m: m \leqq\left(b_{n}-a_{n}\right) \mathcal{P}_{n} \quad \text { and } \quad p_{b_{n}-m} P\left(\left|X_{n}-X\right| \geqq \epsilon\right) \geqq \delta\right\} \subseteq \mathcal{K},
$$

where

$$
\mathcal{K}=\left\{m: m \leqq\left(b_{n}-a_{n}\right) \mathcal{P}_{n} \quad \text { and } \quad p_{b_{n}-m}\left|x_{m}-L\right| \geqq \epsilon\right\} .
$$

Thus, by Definition 2, we may write

$$
\text { stat }_{\mathrm{D}(\mathrm{CN}) \mathrm{P}} X_{n} \rightarrow X .
$$

We now present below an example to show that a sequence of random variables is deferred Cesàro and deferred Nörlund $[\mathrm{D}(\mathrm{CN}) \mathrm{P}]$ statistically probability convergent, whenever it is not deferred Cesàro and deferred Nörlund $[\mathrm{D}(\mathrm{CN})]$ statistically convergent. 
Example 2. Let $a_{n}=2 n-1, b_{n}=4 n-1$ and $p_{n}=n$. Suppose that the probability density function of $X_{n}$ is given by

$$
f_{n}(x)= \begin{cases}1 & \left(0<x<1 ; n=m^{2} \forall m \in \mathbb{N}\right) \\ 0 & \text { (otherwise) }\end{cases}
$$

and

$$
f_{n}(x)= \begin{cases}\frac{(n+1) x^{n}}{4^{n+1}} & \left(0<x<4 ; n \neq m^{2} \forall m \in \mathbb{N}\right) \\ 0 & \text { (otherwise). }\end{cases}
$$

Let $0<\epsilon, \delta<1$. Then

$$
P\left(\left|X_{n}-4\right| \geqq \epsilon\right)= \begin{cases}1 & \text { when } n=m^{2} \\ 1-P\left(\left|X_{n}-4\right|<\epsilon\right)=\left(1-\frac{\epsilon}{4}\right)^{n+1} & \text { when } n \neq m^{2} .\end{cases}
$$

Consequently, we have

$$
\lim _{n \rightarrow \infty} \frac{1}{2 n^{2}} \mid\left\{m: m \leqq 2 n^{2} \quad \text { and } \quad n P\left(\left|X_{n}-4\right| \geqq \epsilon\right) \geqq \delta\right\} \mid=0,
$$

Clearly, we observe that $\left(X_{n}\right)$ is neither convergent nor deferred Cesàro and deferred Nörlund $[\mathrm{D}(\mathrm{CN})]$ statistically convergent; however, it is deferred Cesàro and deferred Nörlund $[\mathrm{D}(\mathrm{CN}) \mathrm{P}]$ statistically probability convergent to 4 .

\section{A NEW KOROVKIN-TYPE THEOREM}

In this section, we extend here the result of Jena et al. [8] and Srivastava et al. [24] by using the product deferred Cesàro and deferred Nörlund [D(CN)P] statistical probability convergence of sequences of random variables over a Banach space.

Let $\mathcal{C}(\mathbb{R})$, be the space of all real valued continuous probability functions defined on $\mathbb{R}$ under the norm $\|\cdot\|_{\infty}$. Also let $\mathcal{C}(\mathbb{R})$ is a Banach space. Then, for $f \in \mathcal{C}(\mathbb{R})$, the norm of $f$ denoted by $\|f\|$ is given by,

$$
\|f\|_{\infty}=\sup _{x \in \mathbb{R}}\{|f(x)|\} .
$$

We say that the operator $\mathfrak{L}$ is a sequence of random variables of positive linear operator provided that

$$
\mathfrak{L}(f ; x) \geqq 0 \quad \text { whenever } \quad f \geqq 0 .
$$

Now we prove the following theorem by using the product deferred Cesàro and deferred Nörlund $[\mathrm{D}(\mathrm{CN}) \mathrm{P}]$ statistical probability convergence. 
Theorem 2. Let

$$
\mathfrak{L}_{m}: \mathcal{\ell}(\mathbb{R}) \rightarrow \mathcal{C}(\mathbb{R})
$$

be a sequence of random variables of positive linear operators. Then, for all $f \in$ $e(\mathbb{R})$

$$
\operatorname{stat}_{\mathrm{D}(\mathrm{CN}) \mathrm{P}} \lim _{m \rightarrow \infty}\left\|\mathfrak{L}_{m}(f ; x)-f(x)\right\|_{\infty}=0
$$

if and only if

$$
\begin{aligned}
& \operatorname{stat}_{\mathrm{D}(\mathrm{CN}) \mathrm{P}} \lim _{m \rightarrow \infty}\left\|\mathfrak{L}_{m}(1 ; x)-1\right\|_{\infty}=0, \\
& \operatorname{stat}_{\mathrm{D}(\mathrm{CN}) \mathrm{P}} \lim _{m \rightarrow \infty}\left\|\mathfrak{L}_{m}(x ; x)-x\right\|_{\infty}=0,
\end{aligned}
$$

and

$$
\operatorname{stat}_{\mathrm{D}(\mathrm{CN}) \mathrm{P}} \lim _{m \rightarrow \infty}\left\|\mathfrak{L}_{m}\left(x^{2} ; x\right)-x^{2}\right\|_{\infty}=0 .
$$

Proof. Since each of the following functions

$$
f_{0}(x)=1, \quad f_{1}(x)=x \quad \text { and } \quad f_{2}(x)=x^{2}
$$

belonging to $\mathcal{C}(\mathbb{R})$ and are continuous, the implication given by (3.1) implies (3.2) to (3.4) is obvious. In order to complete the proof of the Theorem 2, we first assume that the conditions (3.2) to (3.4) hold true. If $f \in \mathscr{C}(\mathbb{R})$, then there exists a constant $\mathcal{M}>0$ such that

$$
|f(x)| \leqq \mathcal{M} \quad(\forall x \in \mathbb{R}) .
$$

We thus find that

$$
|f(s)-f(x)| \leqq 2 \mathcal{M} \quad(s, x \in I) .
$$

Clearly, for given $\epsilon>0$, there exists $\delta>0$ such that

$$
|f(s)-f(x)|<\epsilon
$$

whenever

$$
|s-x|<\delta, \quad \text { for alls, } x \in I \text {. }
$$

Let us choose

$$
\varphi_{1}=\varphi_{1}(s, x)=(s-x)^{2} .
$$

If $|s-x| \geqq \delta$, then we obtain

$$
|f(s)-f(x)|<\frac{2 \mathcal{M}}{\delta^{2}} \varphi_{1}(s, x) .
$$

From equation (3.6) and (3.7), we get

$$
|f(s)-f(x)|<\epsilon+\frac{2 \mathcal{M}}{\delta^{2}} \varphi_{1}(s, x),
$$


which implies that

$$
-\epsilon-\frac{2 \mathcal{M}}{\delta^{2}} \varphi_{1}(s, x) \leqq f(s)-f(x) \leqq \epsilon+\frac{2 \mathcal{M}}{\delta^{2}} \varphi_{1}(s, x) .
$$

Now since $\mathfrak{L}_{m}(1 ; x)$ is monotone and linear, so by applying the operator $\mathfrak{L}_{m}(1 ; x)$ to this inequality, we have

$$
\begin{aligned}
\mathfrak{L}_{m}(1 ; x)\left(-\epsilon-\frac{2 \mathcal{M}}{\delta^{2}} \varphi_{1}(s, x)\right) & \leqq \mathfrak{L}_{m}(1 ; x)(f(s)-f(x)) \\
& \leqq \mathfrak{L}_{m}(1 ; x)\left(\epsilon+\frac{2 \mathcal{M}}{\delta^{2}} \varphi_{1}(s, x)\right) .
\end{aligned}
$$

We note that $x$ is fixed and so $f(x)$ is a constant number. Therefore, we have

$$
\begin{aligned}
-\epsilon \mathfrak{L}_{m}(1 ; x)-\frac{2 \mathcal{M}}{\delta^{2}} \mathfrak{L}_{m}\left(\varphi_{1} ; x\right) & \leqq \mathfrak{L}_{m}(f ; x)-f(x) \mathfrak{L}_{m}(1 ; x) \\
& \leqq \epsilon \mathfrak{L}_{m}(1 ; x)+\frac{2 \mathcal{M}}{\delta^{2}} \mathfrak{L}_{m}\left(\varphi_{1} ; x\right) .
\end{aligned}
$$

But

$$
\mathfrak{L}_{m}(f ; x)-f(x)=\left[\mathfrak{L}_{m}(f ; x)-f(x) \mathfrak{L}_{m}(1 ; x)\right]+f(x)\left[\mathfrak{L}_{m}(1 ; x)-1\right] .
$$

Using (3.9) and (3.10), we have

$$
\mathfrak{L}_{m}(f ; x)-f(x)<\epsilon \mathfrak{L}_{m}(1 ; x)+\frac{2 \mathcal{M}}{\delta^{2}} \mathfrak{L}_{m}\left(\varphi_{1} ; x\right)+f(x)\left[\mathfrak{L}_{m}(1 ; x)-1\right] .
$$

We now estimate $\mathfrak{L}_{m}\left(\varphi_{1} ; x\right)$ as follows:

$$
\begin{aligned}
\mathfrak{L}_{m}\left(\varphi_{1} ; x\right)= & \mathfrak{L}_{m}\left((s-x)^{2} ; x\right)=\mathfrak{L}_{m}\left(s^{2}-2 x s+x^{2} ; x\right) \\
= & \mathfrak{L}_{m}\left(s^{2} ; x\right)-2 x \mathfrak{L}_{m}(s ; x)+x^{2} \mathfrak{L}_{m}(1 ; x) \\
= & {\left[\mathfrak{L}_{m}\left(s^{2} ; x\right)-x^{2}\right]-2 x\left[\mathfrak{L}_{m}(s ; x)-x\right] } \\
& \quad+x^{2}\left[\mathfrak{L}_{m}(1 ; x)-1\right] .
\end{aligned}
$$

Using (3.11), we obtain

$$
\begin{aligned}
\mathfrak{L}_{m}(f ; x)-f(x)<\epsilon & \mathfrak{L}_{m}(1 ; x)+\frac{2 \mathcal{M}}{\delta^{2}}\left\{\left[\mathfrak{L}_{m}\left(s^{2} ; x\right)-x^{2}\right]\right. \\
& \left.-2 x\left[\mathfrak{L}_{m}(s ; x)-e^{-x}\right]+x^{2}\left[\mathfrak{L}_{m}(1 ; x)-1\right]\right\} \\
& +f(x)\left[\mathfrak{L}_{m}(1 ; x)-1\right] . \\
=\epsilon & {\left[\mathfrak{L}_{m}(1 ; x)-1\right]+\epsilon+\frac{2 \mathcal{M}}{\delta^{2}}\left\{\left[\mathfrak{L}_{m}\left(s^{2} ; x\right)-x^{2}\right]\right.} \\
& \left.-2 x\left[\mathfrak{L}_{m}(s ; x)-x\right]+x^{2}\left[\mathfrak{L}_{m}(1 ; x)-1\right]\right\} \\
& +f(x)\left[L_{m}(1 ; x)-1\right] .
\end{aligned}
$$


Since $\epsilon>0$ is arbitrary, we can write

$$
\begin{aligned}
\left|\mathfrak{L}_{m}(f ; x)-f(x)\right| \leqq & +\left(\epsilon+\frac{2 \mathcal{M}}{\delta^{2}}+\mathcal{M}\right)\left|\mathfrak{L}_{m}(1 ; x)-1\right| \\
+ & \frac{4 \mathcal{M}}{\delta^{2}}\left|\mathfrak{L}_{m}(s ; x)-x\right|+\frac{2 \mathcal{M}}{\delta^{2}}\left|\mathfrak{L}_{m}\left(s^{2} ; x\right)-x^{2}\right| \\
\leqq & \mathcal{K}\left(\left|\mathfrak{L}_{m}(1 ; x)-1\right|+\left|\mathfrak{L}_{m}(s ; x)-x\right|\right. \\
& \left.\quad+\left|\mathfrak{L}_{m}\left(s^{2} ; x\right)-x^{2}\right|\right)
\end{aligned}
$$

where

$$
\mathcal{K}=\max \left(\epsilon+\frac{2 \mathcal{M}}{\delta^{2}}+\mathcal{M}, \frac{4 \mathcal{M}}{\delta^{2}}, \frac{2 \mathcal{M}}{\delta^{2}}\right) .
$$

Now, for a given $r>0$, there exists $\delta, \epsilon>0$, such that $\epsilon<r$. Then, by setting

$$
\Theta_{m}(x ; r)=\left\{m: m \leqq\left(b_{n}-a_{n}\right) \mathcal{P}_{n} \text { and } p_{b_{n}-m} P\left(\left|\mathfrak{L}_{m}(f ; x)-f(x)\right| \geqq r\right)\right\} \geqq \delta \text {. }
$$

Also, for $i=0,1,2$, we have

$$
\begin{gathered}
\Theta_{i, m}(x ; r)=\left\{m: m \leqq\left(b_{n}-a_{n}\right) \mathcal{P}_{n}\right. \\
\text { and } \left.\quad p_{b_{n}-m} P\left(\left|\mathfrak{L}_{m}\left(f_{i} ; x\right)-f_{i}(x)\right| \geqq \frac{r-\epsilon}{3 \mathcal{K}}\right)\right\} \geqq \delta,
\end{gathered}
$$

so that,

$$
\Theta_{m}(x ; r) \leqq \sum_{i=0}^{2} \Theta_{i, m}(x ; r) .
$$

Clearly, we have

$$
\frac{\left\|\Theta_{m}(x ; r)\right\| \mathcal{e}(\mathbb{R})}{\left(b_{n}-a_{n}\right) \mathcal{P}_{n}} \leqq \sum_{i=0}^{2} \frac{\left\|\Theta_{i, m}(x ; r)\right\| \mathcal{e}(\mathbb{R})}{\left(b_{n}-a_{n}\right) \mathcal{P}_{n}} .
$$

Now, using the above assumption about the implications in (3.2) to (3.4) and by Definition 2, the right-hand side of (3.13) is seen to tend to zero as $n \rightarrow \infty$. Consequently, we get

$$
\lim _{n \rightarrow \infty} \frac{\left\|\Theta_{m}(x ; r)\right\|_{\mathcal{C}(\mathbb{R})}}{\left(b_{n}-a_{n}\right) \mathcal{P}_{n}}=0(\delta, r>0) .
$$

Therefore, the implication (3.1) holds true. This completes the proof of Theorem 2.

Now, by using the Definition 1, we present the following corollary as the consequence of Theorem 2.

Corollary 1. Let $\mathfrak{L}_{m}: \mathcal{C}(\mathbb{R}) \rightarrow \mathcal{C}(\mathbb{R})$ be a sequence of positive linear operators. Also let $f \in \mathcal{C}(\mathbb{R})$. Then

$$
\operatorname{stat}_{\mathrm{D}(\mathrm{CN})} \lim _{m \rightarrow \infty}\left\|\mathfrak{L}_{m}(f ; x)-f(x)\right\|_{\infty}=0
$$


if and only if

$$
\begin{aligned}
& \operatorname{stat}_{\mathrm{D}(\mathrm{CN})} \lim _{m \rightarrow \infty}\left\|\mathfrak{L}_{m}(1 ; x)-1\right\|_{\infty}=0, \\
& \operatorname{stat}_{\mathrm{D}(\mathrm{CN})} \lim _{m \rightarrow \infty}\left\|\mathfrak{L}_{m}(x ; x)-x\right\|_{\infty}=0,
\end{aligned}
$$

and

$$
\operatorname{stat}_{\mathrm{D}(\mathrm{CN})} \lim _{m \rightarrow \infty}\left\|\mathfrak{L}_{m}\left(x^{2} ; x\right)-x^{2}\right\|_{\infty}=0 .
$$

We present below an illustrative example for the sequence of random variables of positive linear operators that does not satisfy the conditions of the Korovkin approximation theorems proved earlier by Jena et al. [8], Srivastava et al. [24] and Paikray et al. [15], but which satisfies the conditions of our Theorem 2. Consequently, our Theorem 2 is stronger than the results established earlier by both Jena et al. [8] and Srivastava et al. [24].

We now recall the operator

$$
x(1+x D) \quad\left(D=\frac{d}{d x}\right)
$$

which was used by Al-Salam [2] and, more recently, by Viskov and Srivastava [28] (see also [18] the monograph by Srivastava and Manocha [26] for various general families of operators of this kind). Here, in our Example 3 below, we use this operator in conjunction with the Meyer-König and Zeller operators.

Example 3. Let $X=[0,1]$ and we consider Meyer-König and Zeller operators $\mathfrak{M}_{n}(f ; x)$ on $\mathcal{C}[0,1]$ given by (see [3]),

$$
\mathfrak{M}_{n}(f ; x)=\sum_{k=0}^{\infty} f\left(\frac{k}{k+n+1}\right)\left(\begin{array}{c}
n+k \\
k
\end{array}\right) x^{k} .(1+x)^{n+1} .
$$

Also let $\mathfrak{L}_{m}: \mathcal{C}[0,1] \rightarrow \mathcal{C}[0,1]$ be sequence of operators defined as follows:

$$
\mathfrak{L}_{m}(f ; x)=\left[1+X_{m}\right] x(1+x D) \mathfrak{M}_{m}(f) \quad(f \in \mathcal{C}(\mathbb{R}),
$$

where $\left(X_{m}\right)$ is a sequence of random variables defined in Example 2.

Now,

$$
\begin{gathered}
\mathfrak{L}_{m}(1 ; x)=\left[1+X_{m}\right] x(1+x D) 1=\left[1+X_{m}\right] x, \\
\mathfrak{L}_{m}(s ; x)=\left[1+X_{m}\right] x(1+x D) x=\left[1+X_{m}\right] x(1+x),
\end{gathered}
$$

and

$$
\mathfrak{L}_{m}\left(s^{2} ; x\right)=\left[1+X_{n}\right] x(1+x D)\left\{x^{2}\left(\frac{n+2}{n+1}\right)+\frac{x}{n+1}\right\}
$$




$$
=\left[1+X_{n}(x)\right]\left\{x^{2}\left[\left(\frac{n+2}{n+1}\right) x+2\left(\frac{1}{n+1}\right)+2 x\left(\frac{n+2}{n+1}\right)\right]\right\},
$$

so that we have

$$
\begin{aligned}
& \operatorname{stat}_{\mathrm{D}(\mathrm{CN}) \mathrm{P}} \lim _{m \rightarrow \infty}\left\|\mathfrak{L}_{m}(1 ; x)-1\right\|_{\infty}=0, \\
& \operatorname{stat}_{\mathrm{D}(\mathrm{CN}) \mathrm{P}} \lim _{m \rightarrow \infty}\left\|\mathfrak{L}_{m}(x ; x)-x\right\|_{\infty}=0,
\end{aligned}
$$

and

$$
\operatorname{stat}_{\mathrm{D}(\mathrm{CN}) \mathrm{P}} \lim _{m \rightarrow \infty}\left\|\mathfrak{L}_{m}\left(x^{2} ; x\right)-x^{2}\right\|_{\infty}=0,
$$

that is, the sequence $\mathfrak{L}_{m}(f ; x)$ satisfies the conditions (3.2) to (3.4). Therefore by Theorem 2, we have

$$
\operatorname{stat}_{\mathrm{D}(\mathrm{CN}) \mathrm{P}} \lim _{m \rightarrow \infty}\left\|\mathfrak{L}_{m}(f ; x)-f\right\|_{\infty}=0 .
$$

Hence, it is deferred Cesàro and deferred Nörlund $[\mathrm{D}(\mathrm{CN}) \mathrm{P}]$ statistically probability convergent. However, since $\left(X_{m}\right)$ is neither Cesàro nor Nörlund statistically convergent, so it is neither deferred Cesàro (DC) statistically convergent nor deferred Nörlund (DN) statistically convergent. Thus, we conclude that earlier works in [8] and [24] are not valid for the operators defined by (3.14), where as our Theorem 2 still works for the operators defined by (3.14).

\section{CONCLUding REMARKS AND OBSERVATIONS}

In this concluding section of our investigation, we present several further remarks and observations concerning to various results which we have proved here.

Remark 1. Let $\left(X_{m}\right)_{m \in \mathbb{N}}$ be a sequence of random variables given in Example 2. Then, since

$$
\operatorname{stat}_{\mathrm{D}(\mathrm{CN}) \mathrm{P}} \lim _{m \rightarrow \infty} X_{m}=4 \text { on }[0,1]
$$

we have

$$
\operatorname{stat}_{\mathrm{D}(\mathrm{CN}) \mathrm{P}} \lim _{m \rightarrow \infty}\left\|\mathfrak{L}_{m}\left(f_{i} ; x\right)-f_{i}(x)\right\|_{\infty}=0 \quad(i=0,1,2) .
$$

Thus, by applying by Theorem 2, we can write

$$
\operatorname{stat}_{\mathrm{D}(\mathrm{CN}) \mathrm{P}} \lim _{m \rightarrow \infty}\left\|\mathfrak{L}_{m}(f ; x)-f(x)\right\|_{\infty}=0, \quad(i=0,1,2),
$$

where

$$
f_{0}(x)=1, \quad f_{1}(x)=x \quad \text { and } \quad f_{2}(x)=x^{2} .
$$

However, since $\left(X_{m}\right)$ is neither statistically convergent nor converges uniformly in the ordinary sense, thus, the classical and statistical Korovkin-type theorems do not work here for the operators defined by (3.14). Hence, clearly, this application indicates that our Theorem 2 is a non-trivial generalization of the classical as well as statistical Korovkin-type theorems (see [6] and [11]). 
Remark 2. Let $\left(X_{m}\right)_{m \in \mathbb{N}}$ be a sequence of random variables as given in Example 2. Then, since

$$
\text { stat }_{\mathrm{D}(\mathrm{CN}) \mathrm{P}} \lim _{m \rightarrow \infty} X_{m}=4 \text { on }[0,1],
$$

so (4.1) holds true. Now by applying (4.1) and Theorem 2, the condition (4.2) holds true. However, since the sequence $\left(X_{m}\right)$ of random variables is not deferred Cesàro [8] and deferred Nörlund statistically convergent, the results of Jena et al. [8] and Srivastava et al. (see [24]) do not work for our operator defined in (3.14). Thus, naturally, our Theorem 2 is also a non-trivial extension of the result of Jena et al. [8] and Srivastava et al. [24] (see also [15] and [17]). Based upon the above results, it is concluded here that our proposed method has successfully worked for the operators defined in (3.14) and, therefore, it is stronger than the classical and statistical versions of the Korovkin-type approximation theorems (see [15], [17] and [24]) which were established earlier.

\section{REFERENCES}

[1] R. P. Agnew, "On deferred Cesàro means." Ann. Math., vol. 33, pp. 413-421, 1932, doi: $10.2307 / 1968524$.

[2] W. A. Al-Salam, "Operational representations for the Laguerre and other polynomials." Duke Math. J., vol. 31, pp. 127-142, 1964, doi: 10.1215/S0012-7094-64-03113-8.

[3] A. Altın, O. Doğru, and F. Taşdelen, "The generalization of Meyer-König and Zeller operators by generating functions.” J. Math. Anal. Appl., vol. 312, pp. 181-194, 2005, doi: 10.1016/j.jmaa.2005.03.086.

[4] A. A. Das, B. B. Jena, S. K. Paikray, and R. K. Jati, "Statistical deferred weighted summability and associated Korovokin-type approximation theorem.” Nonlinear Sci. Lett. A, vol. 9, pp. 238-245, 2018.

[5] H. Dutta, S. K. Paikray, and B. B. Jena, Current Trends in Mathematical Analysis and Its Interdisciplinary Applications: On statistical deferred Cesàro summability. Switzerland AG: Springer, 2019. doi: 10.1007/978-3-030-15242-0_23.

[6] H. Fast, "Sur la convergence statistique." Colloq. Math., vol. 2, pp. 241-244, 1951.

[7] B. B. Jena, S. K. Paikray, and U. K. Misra, "Inclusion theorems on general convergence and statistical convergence of $(L, 1,1)$-summability using generalized Tauberian conditions." Tamsui Oxford J. Inform. Math. Sci., vol. 31, pp. 101-115, 2017.

[8] B. B. Jena, S. K. Paikray, and U. K. Misra, "Statistical deferred Cesàro summability and its applications to approximation theorems." Filomat, vol. 32, pp. 2307-2319, 2018, doi: 10.2298/FIL1806307J.

[9] U. Kadak, N. L. Braha, and H. M. Srivastava, "Statistical weighted $\mathscr{B}$-summability and its applications to approximation theorems." Appl. Math. Comput., vol. 302, pp. 80-96, 2017, doi: 10.1016/j.amc.2017.01.011.

[10] V. Karakaya and T. A. Chishti, "Weighted statistical convergence." Iranian. J. Sci. Technol. Trans. A, vol. 33, pp. 219-223, 2009, doi: 10.22099/IJSTS.2009.2217.

[11] P. P. Korovkin, "Convergence of linear positive operators in the spaces of continuous functions (in Russian).” Doklady Akad. Nauk. SSSR (New Ser.), vol. 90, pp. 961-964, 1953.

[12] S. A. Mohiuddine, A. Alotaibi, and M. Mursaleen, "Statistical summability $(C, 1)$ and a Korovkintype approximation theorem." J. Inequal. Appl., vol. 2012, pp. 1-8, 2012. 
[13] F. Móricz, "Tauberian conditions under which statistical convergence follows from statistical summability $(C, 1)$.” J. Math. Anal. Appl., vol. 275, pp. 277-287, 2002, doi: 10.1016/S0022247X(02)00338-4.

[14] M. Mursaleen, V. Karakaya, M. Ertürk, and F. Gürsoy, "Weighted statistical convergence and its application to Korovkin-type approximation theorem.” Appl. Math. Comput., vol. 218, pp. 9132 9137, 2012, doi: 10.1016/j.amc.2012.02.068.

[15] S. K. Paikray and H. Dutta, Applied Mathematical Analysis: Theory, Methods, and Applications: On statistical deferred weighted $B$-convergence. Switzerland AG: Springer, 2019.

[16] S. K. Paikray, B. B. Jena, and U. K. Misra, Advances in Summability and Approximation Theory: Statistical deferred Cesàro summability mean based on $(p, q)$-integers with application to approximation theorems. Singapore: Springer, 2019. doi: 10.1007/978-981-13-3077-3_13.

[17] T. Pradhan, S. K. Paikray, B. B. Jena, and H. Dutta, "Statistical deferred weighted $\mathscr{B}$-summability and its applications to associated approximation theorems." J. Inequal. Appl., vol. 2018, pp. 1-21, 2018.

[18] H. M. Srivastava, "A note on certain operational representations for the Laguerre polynomials." $J$. Math. Anal. Appl., vol. 138, pp. 209-213, 1989, doi: 10.1016/0022-247X(89)90331-4.

[19] H. M. Srivastava and M. Et, "Lacunary statistical convergence and strongly lacunary summable functions of order $\alpha . "$ Filomat, vol. 31, pp. 1573-1582, 2017, doi: 10.2298/FIL1706573S.

[20] H. M. Srivastava, B. B. Jena, and S. K. Paikray, "A certain class of statistical probability convergence and its applications to approximation theorems." Appl. Anal. Discrete Math. (in press), pp. $1-18,2019$.

[21] H. M. Srivastava, B. B. Jena, and S. K. Paikray, "Deferred Cesàro statistical probability convergence and its applications to approximation theorems." J. Nonlinear Convex Anal., vol. 20, pp. $1-16,2019$.

[22] H. M. Srivastava, B. B. Jena, S. K. Paikray, and U. K. Misra, "A certain class of weighted statistical convergence and associated Korovkin type approximation theorems for trigonometric functions." Math. Methods Appl. Sci., vol. 41, pp. 671-683, 2018, doi: 10.1002/mma.4636.

[23] H. M. Srivastava, B. B. Jena, S. K. Paikray, and U. K. Misra, "Deferred weighted A-A-statistical convergence based upon the $(p, q)$-Lagrange polynomials and its applications to approximation theorems." J. Appl. Anal., vol. 24, pp. 1-16, 2018, doi: 10.1515/jaa-2018-0001.

[24] H. M. Srivastava, B. B. Jena, S. K. Paikray, and U. K. Misra, "Generalized equi-statistical convergence of the deferred Nörlund summability and its applications to associated approximation theorems." Rev. R. Acad. Cienc. Exactas Fís. Nat. Ser. A Mat. (RACSAM), vol. 112, pp. 14871501, 2018, doi: 10.1007/s13398-017-0442-3.

[25] H. M. Srivastava, B. B. Jena, S. K. Paikray, and U. K. Misra, "Statistically and relatively modular deferred-weighted summability and Korovkin-type approximation theorems." Symmetry, vol. 11, pp. 1-20, 2019, doi: 10.3390/sym11040448.

[26] H. M. Srivastava and H. L. Manocha, A Treatise on Generating Functions. New York, Chichester, Brisbane and Toronto: John Wiley and Sons, 1984.

[27] H. Steinhaus, "Sur la convergence ordinaire et la convergence asymptotique." Colloq. Math., vol. 2, pp. 73-74, 1951.

[28] O. V. Viskov and H. M. Srivastava, "New approaches to certain identities involving differential operators.” J. Math. Anal. Appl., vol. 186, pp. 1-10, 1994, doi: 10.1006/jmaa.1994.1281. 
Authors' addresses

Bidu Bhusan Jena

Department of Mathematics, Veer Surendra Sai University of Technology, Burla 768018, Odisha, India

E-mail address: bidumath.05@gmail.com

\section{Susanta Kumar Paikray}

Department of Mathematics, Veer Surendra Sai University of Technology, Burla 768018, Odisha, India

E-mail address: skpaikray_mathevssut.ac.in 